\title{
The brain knows the difference: two types of grammatical violations
}

\author{
Angela D. Friederici ${ }^{\mathrm{a}, *}$, Martin Meyer ${ }^{\mathrm{a}, \mathrm{b}}$ \\ ${ }^{a}$ Max Planck Institute of Cognitive Neuroscience, P.O. Box 500 355, 04303 Leipzig, Germany \\ ${ }^{\mathrm{b}}$ Department of Neuropsychology, Institute of Psychology, University of Zurich, Zurich, Switzerland
}

Accepted 30 October 2003

\begin{abstract}
The brain has been shown to honor the fundamental linguistic difference between semantic and syntactic information. Here we demonstrate that it even further indicates the necessity to distinguish between two differential syntactic processes: that is to say between the processing of phrase structure information necessary to build up syntactic structures on-line and verb argument structure information crucial to build up representations of who is doing what to whom. The former process is reflected in the event-related brain potentials (ERPs) as an anterior negativity followed by a late centro-parietal positivity, whereas the latter process is reflected as a centro-parietal negativity-positivity pattern. The different ERP patterns clearly suggest that the theoretically assumed difference between local syntactic structure building and argument structure processing is neurophysiologically real.
\end{abstract}

(C) 2004 Elsevier B.V. All rights reserved.

Theme: Neural basis of behavior

Topic: Cognition

Keywords: Sentence processing; Syntax; Event-related brain potentials; N400; P600

\section{Introduction}

Sentence comprehension requires the on-line processing of different kinds of linguistic information available in inflowing language; namely building up a phonological representation and decoding syntactic, lexical, semantic, and pragmatic cues. At least, these various information types have to be integrated to achieve a proper sentence interpretation. Grammatical violations and semantic incongruencies cause problems in accomplishing sentence comprehension (parsing) and sometimes even require a revision of the sentence structure, sentence meaning, or the relation between these levels of linguistic analysis.

A growing number of studies report specific eventrelated brain potentials (ERP), components for different aspects of language processing. Generally, ERP responses are taken to investigate how the brain responds to linguistic anomalies as ERP parameters (amplitude, latency) vary in accord with grammatical violations and semantic incon\footnotetext{
113.

* Corresponding author. Tel.: +49-341-9940-111; fax: +49-341-9940-

E-mail address: angelafr@ens.mpg.de (A.D. Friederici).
}

gruencies. Recent studies have identified a specific component for the processing of lexical-semantic aspects, namely a centro-parietally distributed negativity around $400 \mathrm{~ms}$ post word onset $[17,18]$. This component has been labeled N400. It is observed as a function of lexical-semantic integration problems. Differential aspects of syntactic processing have been correlated with an early and a late ERP component, namely a left anterior negativity (LAN) between 100 and $500 \mathrm{~ms}$ and a centro-parietal positivity post $600 \mathrm{~ms}$ (P600). Morphosyntactic violations such as subject-verb agreement evoke a LAN between 300 and $500 \mathrm{~ms}$ followed by a P600 $([1,11,20]$, but see Ref. [24]). Phrase structure violations appear to elicit an early LAN (ELAN) followed by a P600 $[8,14,21]$. The latency of the early effect, however, varies as a function of how fast word category information, crucial for local phrase structure building, becomes available. Two factors are of relevance here, first the particular structure of the critical word and second the input conditions. When the structure of the critical word marks word category information in the prefix, this information will become available early during auditory presentation. Under these circumstances, the effect is present early, i.e. 120-200 ms post word onset $[8,14]$. When word category information is marked in the suffix, the crucial information becomes available much 
later with respect to the word onset, but still early with respect to the word category decision point (e.g. refined versus refinement) [7]. Second, input conditions appear to effect the latency of the early LAN as well. While the effect is observed early (when word category information is available early) in the auditory domain $[8,14]$, the effect (for the same stimulus material) is present in the visual domain early only when words are presented fast and under optimal visual input conditions (high contrast), but not when presented under low visual contrast conditions [12] or when presented in a word-by-word fashion with longer pauses between each word [20]. Under the latter two conditions, the effect is reported to be present beyond $300 \mathrm{~ms}$.

The early syntactic negativity observed in correlation with the processing of syntactic phrase structure incongruencies has often been reported to be lateralized to the left hemisphere in the auditory $[7,8,14]$ as well as in the visual domain [7,12,21]. Some studies investigating the processing of phrase structure violations, however, found the early anterior negativity more bilaterally distributed [16]. The LAN reflecting morphosyntactic violations usually demonstrates a clear left lateralization $[1,4]$. The late centro-parietal positivity (P600) has been described in association with a wide range of different syntactic anomalies including those requiring a reanalysis of the preceding structure $[13,22,23]$ and those requiring a repair of a syntactic violation $[7,8,14,20,21,24]$.

The different syntactic ERP effects have been taken to reflect different stages of syntactic processing during sentence parsing. The ELAN is viewed to reflect the stage of first-pass parsing during which an initial local syntactic structure is built on the basis of word category information, the LAN is taken to reflect syntactic processes in a second stage during which structural and thematic relations are assigned, and the P600 is assumed to reflect processes of syntactic repair and reanalysis. This view is built on a number of converging findings using different languages as discussed in more detail in Ref. [5].

A few language ERP studies have aimed at investigating the processing of verb argument structure information. One of these studies manipulated the subcategorization properties associated with the matrix verb and observed a P600 as a function of a violation of these [25]. Interestingly, this P600 was preceded by an N400-like negativity which, however, did not reach statistical significance. More recently, violations of the syntactic type of the argument (direct versus indirect object) were found to elicit a LAN-P600 pattern and violations of the incorrect number of arguments to elicit an N400-P600 pattern [6]. As the N400 is known to reflect difficulties of lexical-semantic integration $[17,18]$, the latter pattern signaled some aspects of lexical integration. The N400-P600 pattern was interpreted to reflect difficulties in integrating this surplus noun phrase (N400) followed by the attempt to syntactically reanalyze the perceived input (P600). Another series of studies suggested that the N400 is tied to problems of thematic hierarchizing [9]. Thus, it appears that local phrase structure building processes emerging at a first processing stage evoke a different ERP pattern than argument structure building processes occurring at a second processing stage. Thus, these findings seem to suggest that verb argument structure is processed differently than phrase structure. In linguistic theorizing, however, it is still an open question whether phrase structure information and argument structure information should be considered to be distinct processing domains or not $[3,10,15]$.

In the present study, we directly compared the processing of two different aspects of syntactic information during sentence comprehension, namely phrase structure information versus argument structure information. The main motivation of the current study was to elucidate the differential brain circuits that constitute differential aspects of sentence parsing. On the basis of previous studies, we predict an anterior negativity possibly with a left lateralization followed by a P600 for the processing of incongruent phrase structure information in sentences carrying a word category violation. For the verb argument structure violations, the ERP pattern should be different as the relevant information to be processed taps a later processing stage, namely the stage at which the information about the verb and its arguments is accessed and mapped onto the initial structure built. These latter processes may be expressed in an N400P600 pattern.

\section{Materials and methods}

The German sentence material designed to evaluate the processing of the two different types of structural information differs only in one element, namely the presence or absence of the complementizer 'dass/that' introducing a complement clause. In the complement clause, we used only verbs which mandatorily required two arguments (subject and object).

Phrase structure violation (PSV)

\begin{tabular}{ll}
\hline (1a) correct: & Er meinte dass Lisa Ärger verursacht. \\
& He mentioned that Lisa trouble causes. \\
(1b) incorrect: & Er meinte auch Lisa Ärger verursacht. \\
& He mentioned also Lisa trouble causes. \\
\hline
\end{tabular}

Argument structure violation (ASV)

\begin{tabular}{ll}
\hline (2a) correct: & Er meinte auch Lisa verursacht Ärger. \\
& He mentioned also Lisa causes trouble. \\
(2b) incorrect: & Er meinte dass Lisa verursacht Ärger. \\
& He mentioned that Lisa causes trouble. \\
\hline
\end{tabular}

Unlike English, the required word order in a German matrix clause and a German complement clause is differential when the latter is introduced by a complementizer: subject-object-verb (1a) instead of subject-verb-object 
like in matrix clauses when there is no complementizer (2a). Therefore, sentence (1b) becomes incorrect at the second noun phrase (underlined) in the complement clause, i.e. the processing system analyzing the inflowing information, called the parser, expects the verb but encounters an object causing a word order violation. Sentence (2b) becomes incorrect at the verb (underlined). Here, the paper encounters a verb which given the complementizer should be the final element of the clause. The verb, however, is a transitive verb, but the object obligatory for transitive verbs is still missing when the verb is encountered resulting in an argument structure violation. Note, that an intransive verb (requiring only one argument, namely the subject Lisa) in this position would render the sentence syntactically correct. Therefore, the phrase structure with an intransitive verb directly following the noun is correct, but a transitive verb type occuring in the same position yields a violation.

In total, 512 sentences were presented: there were 256 unspecific filler sentences containing different syntactic structure. The remaining 256 sentences either contained the complementizer 'dass/that' signaling a complement clause $(n=128)$ or comprised the focus particle 'auch/ also/sogar' signaling a matrix clause $(n=128)$. Half of these sentences were correct $(1 \mathrm{a} / 2 \mathrm{a})$ and half had an incorrect word order resulting either in a phrase structure violation (1b) or in an argument structure violation (2b).

\subsection{Procedure}

Sentences were presented visually one word at a time with a presentation time of $500 \mathrm{~ms}$ and an interstimulus interval of $0 \mathrm{~ms}$. Subjects were instructed to make a grammaticality judgment (yes/no decision) when three centered question marks appeared on the monitor 500 $\mathrm{ms}$ after the sentence offset. Response time was limited to $2000 \mathrm{~ms}$.

\subsection{ERP recordings}

Electrode resistance was kept $<5 \mathrm{~K} \Omega$. Scalp EEG was recorded continuously from 64 channels with a $250 \mathrm{~Hz}$ sampling rate, referenced to the left mastoid. ERP averages were filtered off-line with $10 \mathrm{~Hz}$ low-pass. For detection of eye movements and blink artifacts, a bipolar electrooculogram (EOG) was recorded from two electrodes placed at the outer canthi of the left and the right eye and from two electrodes placed above and below the right eye. All statistical analyses were performed on the mean ERP amplitudes in the different experimental conditions. On the basis of previous studies and visual inspection, we defined three different time windows 380-450 ms (ELAN), and $600-1000 \mathrm{~ms}$ (P600) for the PSV, and 350-600 ms (N400) and 600-1000 ms (P600) for the ASV. In both conditions $t=0 \mathrm{~ms}$ marks the onset of the word which signals a syntactic violation. Due to the different word order in (1) and (2) the critical word is either the noun (PSV) or the verb (ASV).

\subsection{Participants}

Thirty native German-speaking subjects (15 female) participated in the experiment. All participants were righthanded and had normal or corrected to normal vision. Their mean age was 24 years (ranging from 19 to 31 years).

\section{Results}

\subsection{Behavioral data}

No significant effects were found.

\subsection{ERP data}

Different effects were observed for the two types of syntactic violations, as displayed in Figs. 1 and 2. PSV
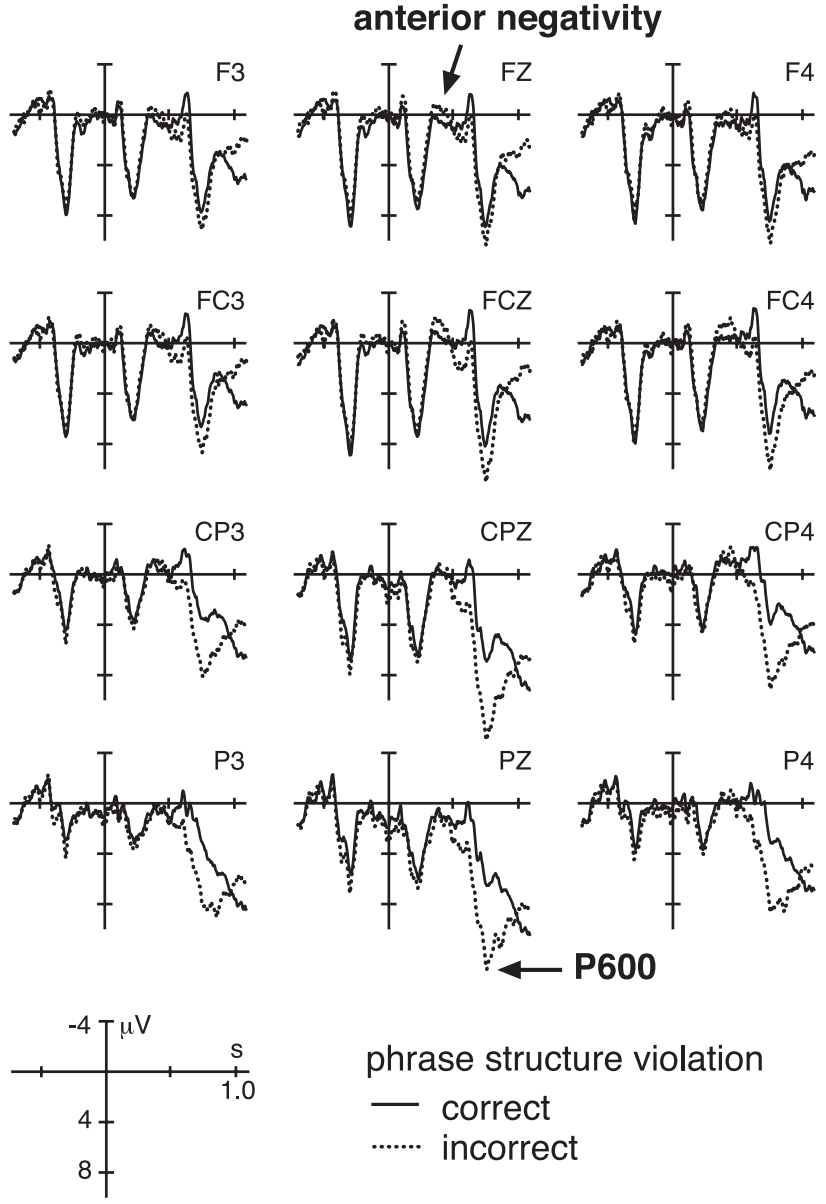

Fig. 1. Averaged ERPs for the phrase structure violation condition. Incorrect: Er meinte auch Lisa Ärger verursacht versus correct: Er meinte dass Lisa Ärger verursacht. The outset of the critical word, i.e. the second noun phrase of the subordinate clause (underlined), is aligned to the zero in the time line. Negativity is plotted up. 
gave rise to an anterior negativity peaking between 380 and $450 \mathrm{~ms}$ followed by a P600 (Fig. 1). ASV, in contrast, elicited an N400, i.e. a centro-parietal negativity manifesting at between 400 and $600 \mathrm{~ms}$ followed by a P600 (Fig. 2).

\subsection{Phase structure violations}

The ANOVA for the PSV was calculated in two different time windows: the P600 time window (600$1000 \mathrm{~ms}$ ) and a small time window (380-450 ms) in which the anterior negativity was observed. Note, significant effects were only found for these time windows. Statistical analysis for all other time windows did not reveal any significant effects.

P600 (600-1000 ms): data from 32 electrodes, with 16 electrodes representing the anterior ROI (FP1, FPZ, FP2, AF3, AFZ, AF4, F5, F3, FZ, F4, F6, FC5, FC3, FCZ, FC4, FC6) and 16 electrodes representing the posterior ROI (CP5, CP3, CPZ, CP4, CP6, P5, P3, PZ, P4, P6, PO3, POZ, PO4, O1, OZ, O2), entered the analysis for the P600 time window. An ANOVA with the factors $\mathrm{ROI} \times$ Condition
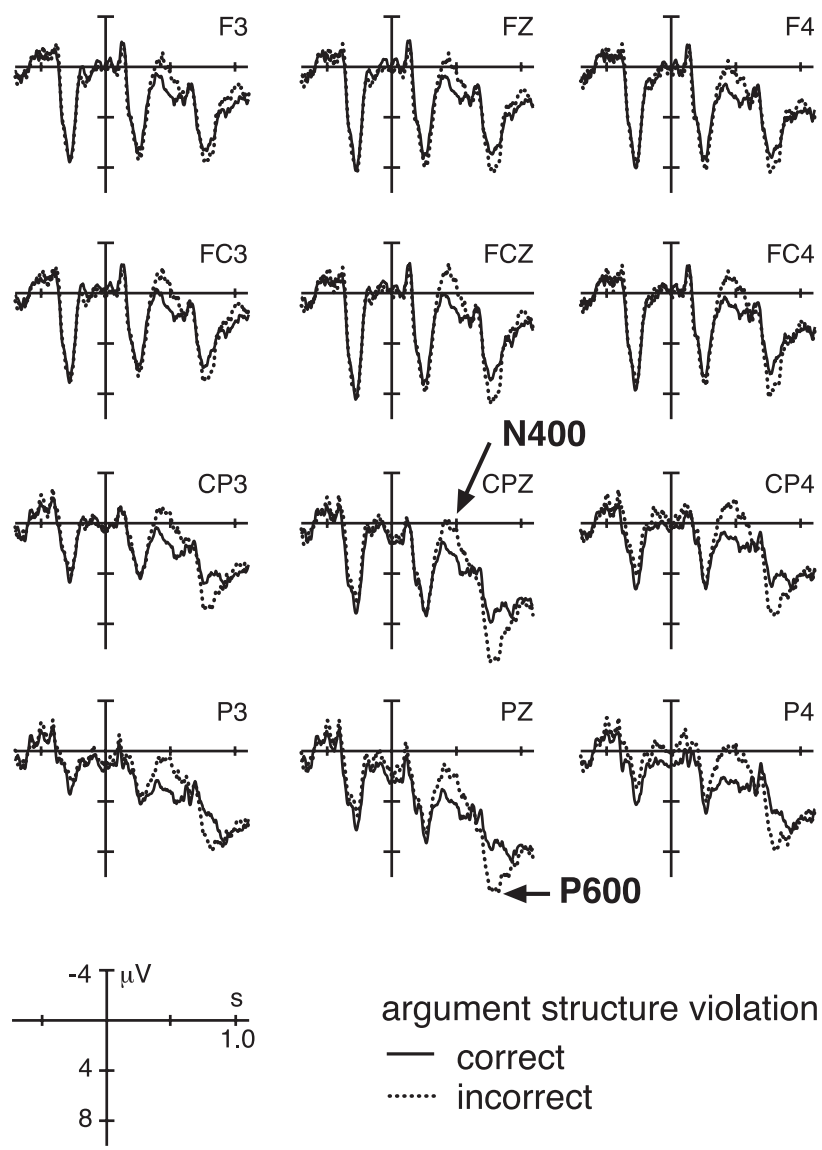

argument structure violation

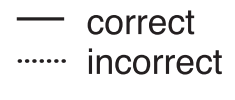

Fig. 2. Averaged ERPs for the argument structure violation condition. Incorrect: Er meinte dass Lisa verursacht Ärger versus correct: Er meinte auch Lisa versursacht Ärger. The outset of the critical word, i.e. verb of the subordinate clause (underlined), is aligned to the zero in the time line. Negativity is plotted up. (correct versus incorrect) revealed a significant interaction for ROI $\times$ Condition $(F(1,29)=18.73, p<0.0005)$. To account for ambiguity effects only normalized raw data using vector length scaling were subjected to ANOVAs with factors ROI [19]. The effect of Condition was significant in the posterior ROI $(F(1,29)=62.56 ; p<0.0001)$, but not in the anterior ROI, indicating that the P600 is maximal over the posterior region. No main effect of Hemisphere or Interaction was found.

Anterior negativity (380-450 ms): data from 20 electrodes, with 10 electrodes representing the anterior ROI (F5, F3, FZ, F4, F6, FC5, FC3, FCZ, FC4, FC6) and 10 electrodes representing the posterior ROI (CP5, CP3, CPZ, CP4, CP6, P5, P3, PZ, P4, P6), entered the analysis for the temporally restricted time window in which the anterior negativity was observed. Statistical analysis revealed a marginally significant $\mathrm{ROI} \times$ Condition interaction $(F(1,29)=3.02, p=0.09)$. The effect of Condition was significant in the anterior ROI $(F(1,29)=4.30$, $p<0.05$ ), but not in the posterior ROI. To test for effects of lateralization ROIs were split up in quadrants allowing the comparison of left versus right scalp electrodes. No main effect of Hemisphere or interaction with this factor was found.

\subsection{Argument structure violation}

The ANOVA for the ASV was calculated for two different time windows: the P600 time window (600$1000 \mathrm{~ms}$ ) and the N400 time window (350-600 ms). Statistical analyses of other time windows prior to and after these critical time windows did not reveal any significant effects. Data from 32 electrodes with 16 electrodes representing the anterior ROI (FP1, FPZ, FP2, AF3, AFZ, AF4, F5, F3, FZ, F4, F6, FC5, FC3, FCZ, FC4, FC6) and 16 electrodes representing the posterior ROI (CP5, CP3, CPZ, CP4, CP6, P5, P3, PZ, P4, P6, PO3, POZ, PO4, O1, OZ, $\mathrm{O} 2)$ entered the analyses for both time windows.

P600 (600-100 ms): the ANOVA with the factors ROI $\times$ Condition revealed a significant interaction $(F(1,29)=7.65, p<0.001)$, indicating a larger effect of Condition in the posterior as compared to the anterior region. The main effect of Condition was significant in the posterior region $(F(1,29)=5.60, p<0.05)$, but not in the anterior region.

N400 (350-600 ms): The ANOVA revealed no significant interaction. A clear effect of Condition was found for the posterior ROI $(F(1,29)=23.78, p<0.0001)$ and for the anterior ROI $(F(1.29)=8.72, p<0.01)$.

\section{Discussion}

The two types of syntactic violations resulted in two different ERP patterns. While the phrase structure violation (PSV) elicited an anterior negativity followed by a 
P600, the argument structure violation (ASV) gave rise to an N400-P600 pattern. These two different ERP patterns for the two types of violations suggest that different brain systems constitute the processes in the early time window, but not those in the late time window. We will discuss the ERP pattern of the PSV and the ASV in turn.

Violations of local phrase structure in earlier studies were reported to evoke an anterior negativity between 100 and $500 \mathrm{~ms}$ followed by a P600 [8,12,21]. In these studies, the anterior negativity was often left lateralized, although it was also observed with a more bilateral distribution in other studies [16]. Most of the studies that found an early left anterior negativity, i.e. around $150 \mathrm{~ms}$, had employed auditory presentations of connected speech and used stimulus material in which word category was marked in the prefix. In the visual modality, the latency of the anterior negativity also varies with the quality of visual input parameters and with the point at which word category information becomes available. The effect is present early when visual input can be processed fast and when the critical target either carries word category information in the prefix [12] or is short altogether (critical word is 'of', Ref. [21]). The present anterior negativity was significant between 380 and $450 \mathrm{~ms}$ which is compatible with previous studies using visual presentation in its temporal structure $[12,20]$. The general pattern of an anterior negativity followed by a P600 is consistent with the vast majority of studies investigating phrase structure violations.

The observed N400-P600 pattern for ASV is in line with an earlier study on verb argument processing in German [6]. In this study, such a pattern was found when the critical verb did not match the number of arguments presented in the previous part of the sentence. The presence of an N400 for verb argument structure violation was interpreted to reflect difficulties of lexical integration due to problems in assigning a role to the surplus argument. In the present study a similar situation arises due to the presence of the complementizer 'dass/ that' in sentence (2b) which signals a complement clause requiring the verb to be in clause final position. When encountering a transitive verb the parser has not yet been delivered with the obligatory number of arguments required by the verb resulting in an N400-P600 pattern. More specifically, the observed N400-P600 in the present study indicates that the parser, having processed the complementizer, has difficulties in integrating the verb as the number of thematically relevant arguments in preverbal position is insufficient. The following P600, meant to reflect processes of syntactic reanalysis or repair, indicates that the parser refers to the syntactic level for revision once it failed to integrate the verb at a thematic level. Complementary to this, an N400-P600 ERP pattern was also recently observed in incorrect German sentences with two arguments, both marked as grammatical subjects [9]. This finding was interpreted to reflect thematic hierarchizing during sentence processing. A recent model that is built on these and related findings postulates parallel syntactic and thematic processing procedures [2]. More specifically, this model assumes that in the presence of morphosyntactic information, which unambiguously marks case, the parser can directly induce the build-up of a thematic hierarchy. The present data indicate that different processes play a role in PSVs and ASVs. Apparently, difficulties of thematic structure building can be triggered by incorrect case marking or an incorrect word order resulting in a situation in which all arguments mandatorily required by the verb are not available when encountering the verb. The combined data from the present and earlier studies indicate that both verbs and noun phrases can elicit an N400-P600 pattern once the number of arguments required by the verb is mismatched during on-line processing. Too many arguments and missing arguments, (Ref. [6], and present study) as well as two arguments of the same type [9] lead to a difficulty in thematic structure building, rendering a match between initial syntactic structure building and the structure required by the type of verb impossible. Therefore, the present study adds to the previous studies in demonstrating that thematic processes as reflected by the N400 can be elicited in the absence of overt case marking which are induced by an insufficient number of arguments available during sentence comprehension.

Here we investigated two types of syntactic incongruities, namely a phrase structure violation and an argument structure violation. The former was purely syntactic in nature whereas the latter resulted in processing difficulties in mapping from syntax to verb argument structure. The phrase structure violation gave rise to an anterior negativity between 380 and $450 \mathrm{~ms}$ followed by a P600. The argument structure violation elicited an N400 with a centro-parietal topography followed by a P600. The differential spatial distribution of the negativities indicates an involvement of distinct brain systems in accomplishing the two violation types. From the observation that distinct ERP responses flagged the different types of grammatical violations, we propose that the detecting and processing of these violations occurs at functionally separate stages during sentence processing. Psycholinguistic and linguistic theorizing might want to consider this neurophysiological difference when modeling syntactic processes.

\section{Acknowledgements}

The work was conducted while Martin Meyer was a research fellow at the Max Planck Institute of Cognitive Neuroscience in Leipzig. We thank Thom Gunter for his assistance in building and programming the design and Juergen Weissenborn for helpful comments on the manuscript. 


\section{References}

[1] A. Angrilli, B. Penolazzi, F. Vespignani, et al, Cortical brain responses to semantic incongruity and syntactic violation in Italian language: an event-related potential study, Neurosci. Lett. 322 (2002) 5-8.

[2] I. Bornkessel (Ed.), The Argument Dependency Model: A Neurocognitive Approach to Incremental Interpretation, MPI Series in Cognitive Neuroscience, vol. 28, Max Planck Institute of Cognitive Neuroscience, Leipzig, 2002.

[3] N. Chomsky, Lectures on Government and Binding, Foris, Dordrecht, 1981.

[4] A. Deutsch, S. Bentin, Syntactic and semantic factors in processing gender agreement in Hebrew: evidence from ERPs and eye movements, J. Mem. Lang. 45 (2001) 200-224.

[5] A.D. Friederici, Towards a neural basis of auditory sentence processing, Trends Cogn. Sci. 6 (2002) 78-84.

[6] A.D. Friederici, S. Frisch, Verb-argument structure processing: the role of verb-specific and argument-specific information, J. Mem. Lang. 43 (2000) 476-507.

[7] A.D. Friederici, E. Pfeifer, A. Hahne, Event-related brain potentials during natural speech processing: effects of semantic, morphological and syntactic violations, Cogn. Brain Res. 1 (1993) 183-192.

[8] A.D. Friederici, A. Hahne, A. Mecklinger, The temporal structure of syntactic parsing: early and late event-related brain potential effects elicited by syntactic anomalies, J. Exper. Psychol., Learn., Mem., Cogn. 22 (1996) 1219-1248.

[9] S. Frisch, M. Schlesewsky, The N400 reflects problems of thematic hierarchizing, NeuroReport 12 (2001) 3391-3394.

[10] A. Goldberg, A Construction Grammar Approach to Argument Structure, University of Chicago Press, Chicago, 1995.

[11] T.C. Gunter, L. Stowe, G. Mulder, When syntax meets semantics, Psychophysiology 34 (1997) 660-676.

[12] T.C. Gunter, A.D. Friederici, A. Hahne, Brain responses during sentence reading: visual input affects central processes, NeuroReport 10 (1999) 3175-3178.
[13] P. Hagoort, C. Brown, J. Groothusen, The syntactic positive shift (SPS) as an ERP measure of syntactic processing, Lang. Cogn. Processes 8 (1993) 439-483.

[14] A. Hahne, A.D. Friederici, Electrophysical evidence for two steps in syntactic analysis: early automatic and late controlled processes, J. Cogn. Neurosci. 11 (1999) 194-205.

[15] R. Jackendoff, Foundations of Language. Brain, Meaning, Grammar, Evolution, Oxford Univ. Press, Oxford, 2002.

[16] T. Knösche, B. Maeß, A.D. Friederici, Processing of syntactic information monitored by brain surface current density mapping based on MEG, Brain Topogr. 12 (1999) 75-87.

[17] M. Kutas, K.D. Federmeier, Electrophysiology reveals semantic memory use in language comprehension, Trends Cogn. Sci. 4 (2000) 463-470.

[18] M. Kutas, S.A. Hillyard, Reading senseless sentences: brain potentials reflect semantic incongruity, Science 207 (1980) 203-205.

[19] G. McCarthy, C.C. Wood, Scalp distributions of event-related potentials: an ambiguity associated with analysis of variance models, Electroencephalogr. Clin. Neurol. 62 (1985) 203-208.

[20] T.F. Münte, H.J. Heinze, G.R. Mangun, Dissociation of brain activity related to syntactic and semantic aspects of language, J. Cogn. Neurosci. 5 (1993) 335-344.

[21] H.J. Neville, J. Nicol, A. Barss, et al, Syntactically based sentence processing classes: evidence from event-related brain potentials, J. Cogn. Neurosci. 3 (1991) 151-165.

[22] L. Osterhout, P.J. Holcomb, Event-related brain potentials elicited by syntactic anomaly, J. Mem. Lang. 31 (1992) 785-804.

[23] L. Osterhout, P.J. Holcomb, Event-related potentials and syntactic anomaly: evidence of anomaly detection during the perception of continuous speech, Lang. Cogn. Processes 8 (1993) 413-437.

[24] L. Osterhout, L.A. Mobley, Event-related brain potentials elicited by failure to agree, J. Mem. Lang. 34 (1995) 739-773.

[25] L. Osterhout, P.J. Holcomb, D.A. Swinney, Brain potentials elicited by garden-path sentences: evidence of the application of verb information during parsing, J. Exper. Psychol., Learn., Mem., Cogn. 20 (1994) 786-803. 